\title{
¿Por qué el mar no se "desparrama" si solo tiene arena alrededor? Indagación en la playa
}

\author{
M Carmen Parrilla, M. Rut Jiménez-Liso y María Martínez-Chico
}

Área de Didáctica de las Ciencias Experimentales. Universidad de Almería. España

[Recibido el 18 de octubre de 2017, aceptado el 30 de octubre de 2017]

En el presente artículo mostramos el diseño de una secuencia de indagación basada en modelos para Primaria para pensar, hacer, hablar y sentir sobre la playa. Esta secuencia surge de la reflexión de una educadora ambiental tras desarrollar el típico activismo de limpieza de costas o juegos motrices sobre biodiversidad, impactos o pesca.

Palabras clave: playa; Indagación; modelos; secuencia de actividades; educación Primaria

\section{Why the sea doesn't escape if it has only got sand holding it? Inquiry at the beach}

In the current paper the design of a teaching sequence through Model-Based Inquiry for Primary School is presented. Primary school students are engaged to think, do, talk, and feel science at the beach. This sequence arises from an environmental educator reflections after participating in the common activism activities of coast-cleaning or games on fishing and its environmental impacts on biodiversity.

Keywords: beach processes; Model-Based Inquiry; teaching sequence; Primary school

Para citar el artículo. Parrilla, M.C., Jiménez-Liso, M.R. y Martínez-Chico, M. (2017). ¿Por qué el mar no se "desparrama" si solo tiene arena alrededor? Indagación en la playa. Ápice. Revista de Educación Científica, 1(2), 38-46. DOI: https://doi.org/10.17979/arec.2017.1.2.3109

Contacto. mrjimene@ual.es mmartinez@ual.es 


\section{Introducción}

Los centros escolares situados próximos a la costa tienen la oportunidad de conectar el currículo de ciencias con actividades en la playa. A menudo estas actividades se centran en salidas litorales (Pujol, 2003; Rebelo, Marques y Costa, 2004) para observar e identificar especies (o sus restos) en la arena o para concienciar sobre la conservación, observando microplásticos presentes en la arena o proponiendo limpiezas de costas.

Esta concepción de la ambientalización del currículo sigue centrándose en "concienciar" y focaliza el currículo "ambiental" en las corrientes naturalistas o conservacionistas, en contenidos reducidos a alguna de las tres $\mathrm{R}$ (reducir, reciclar y reutilizar). . En las actividades de limpieza de costas se centra en reducir los residuos en las zonas "naturales" como las playas, identificando "concienciación" con "activismo". El "activismo" es un enfoque educativo "en el que no hay una reflexión sobre el sentido de las actividades propuestas, cómo formulamos y organizamos los contenidos, el significado de lo que se hace para las personas que participan en dichas actividades, o su eficacia en cuanto al aprendizaje" (Rodríguez y García, 2009, p.24). En el ámbito de la educación científica este enfoque sería una visión reduccionista de la indagación centrada en el "cacharreo" (Couso, 2014; Martínez del Águila, Montoro, Jiménez-Liso, y Gil, 2016) o en la idea que solo observando se van a extraer conclusiones. En palabras de Rodríguez y García (2009, p. 24), "el protagonismo del aprendiz se reduce a una actividad más manipulativa que reflexiva. Se le exige al alumno/a que sea activo, que manipule los elementos de la realidad, que hagan cosas prácticas, pero no se le pide que piense ni reflexione".

El contexto seleccionado en este artículo proviene de una actividad del Ocean Clean Project en el litoral de El Cabo de Gata (Almería) y de la necesidad de transformar estas actividades de observación y manipulativas en actividades de indagación en las que la educación ambiental y la educación científica realmente produzcan el cruce fecundo (Sauvé, 2010).

Para ello, hemos diseñado una secuencia de actividades siguiendo un enfoque de indagación sobre el ecosistema playa para Educación Primaria. Esta secuencia parte de pregunta inicial que engancha al alumnado porque es próxima a ellos, les intriga (no tiene una respuesta directa), sino que les hace pensar y cuyas propuestas o hipótesis deben probar y analizar. La secuencia de indagación está fundamentada en las dificultades de aprendizaje que hemos encontrado en la literatura didáctica.

\section{Enfoque de enseñanza de indagación para educadores ambientales}

Ante los objetivos y las finalidades diferentes, puede parecer que la educación ambiental y la educación científica van por caminos paralelos, sobre todo si comparamos las visiones típicas de ambas considerando las corrientes naturalistas y conservacionistas para un caso y la corriente científica para otro (Sauvé, 2010). Ahijado, Uranga, Vázquez y Yenes (2001) inciden en que la educación ambiental debe aclarar conceptos sobre los procesos que suceden en el entramado de la Naturaleza, facilitar la comprensión y la valoración del impacto de las relaciones entre el hombre, su cultura y los procesos naturales, y sobre todo, se alienta un cambio de valores, actitudes y hábitos que permitan la elaboración de un código de conducta con respecto a las cuestiones relacionadas con el medio ambiente. No podemos afirmar que el conocimiento produzca cambio de conducta (ahí tenemos a muchos médicos que, a pesar de conocer los males del tabaco, siguen fumando), pero sí debemos considerar que el desconocimiento produce desconexión, desafección y, en palabras de Chawla (2006), es 
imprescindible conectar conocimiento racional y emocional para nuestra identificación y vinculación con la Tierra.

Para esta educación científico-ambiental integral proponemos el enfoque de enseñanza por indagación basada en modelos (MBI en sus siglas en inglés, López-Gay, Jiménez-Liso y Martínez-Chico, 2015) donde los participantes se apropian de una pregunta contextualizada (Sanmartí y Márquez, 2017) que les "engancha" y para la que tienen hipótesis o ideas (que pueden expresar con comentarios, dibujos, expresión corporal, etc.) y que sigue con un “¿Cómo lo comprobamos?” para centrar la resolución en la búsqueda de pruebas y en el diseño experimental que produzca resultados cuya interpretación requiera de un modelo para explicar y predecir con el que extraer conclusiones fundamentadas. Para que nada de lo sucedido pase desapercibido para los participantes concluimos el proceso con la autorregulación de lo aprendido y sentido (López-Gay, Jiménez-Liso y Martínez-Chico, 2015).

\section{Contexto y objetivo}

Como hemos señalado en la introducción, nuestra propuesta surge de la reflexión posterior a vivir la limpieza de playas (Ocean Clean Project) en dos centros de Infantil y Primaria de zonas costeras. La actividad comenzaba explicándole a los niños los diferentes tipos de residuos que nos podíamos encontrar en la orilla de la playa y que también estaban en el mar: plásticos procedentes de envases, bolsas..., papel, cristal, cigarrillos, comida, toallitas...etc. Posteriormente les explicaban el tiempo de degradación de cada uno de los diferentes tipos de residuos "para que así se dieran cuenta de lo que tardan en desintegrarse" (en palabras de los monitores del proyecto) y "cómo todo ello afecta a nuestro medio natural, tanto flora como fauna" (Figura 1).

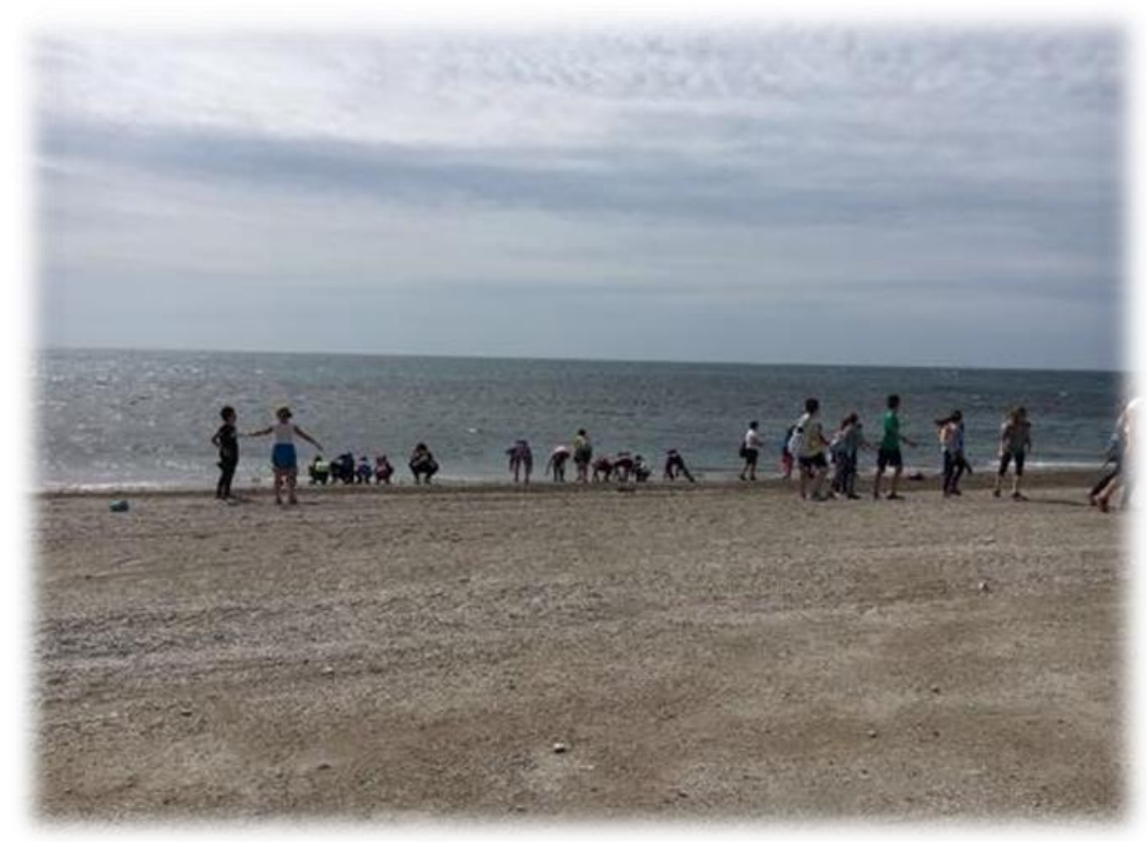

Figura 1. Limpieza de residuos en la playa (Ocean Clean).

Durante el proceso de recogida de residuos hubo que convocar a los niños pues estaban recogiendo restos de posidonia oceánica (planta acuática) como "basura" y hubo que explicarles lo que era la posidonia y su papel tan vital para el ecosistema marino. Tras la 
recogida de residuos en la playa, volvimos al colegio para continuar con juegos relacionados: carrera de la biodiversidad, tarjetas problema/solución, la pesca, el dado de los impactos, canasta de los residuos y la pesca de arrastre.

Tanto los ambientes exteriores al aula como los juegos motrices suelen "motivar al alumnado" en los que se espera que los alumnos aprendan algo (Rebelo, Marques y Costa, 2004). A menudo estas actividades motrices y externas al aula suelen esconder una clase transmisiva camuflada (Pujol, 2003), por ello, con la finalidad de que el alumnado sea consciente de que aprende y de lo que aprende, nos planteamos el siguiente objetivo: Diseñar una secuencia de actividades que siga "al pie de la letra" el enfoque de enseñanza por indagación basada en modelos (MBI, López-Gay, Jiménez-Liso y Martínez-Chico, 2015) sobre y en el ecosistema playa.

Para fundamentar esta secuencia realizamos una revisión de las concepciones alternativas más habituales, teniendo en mente que este contenido está presente en el currículo oficial desde Primaria hasta Bachillerato.

\section{Concepciones alternativas sobre la temática encontrada en la literatura}

La amplia investigación didáctica sobre concepciones alternativas de muchos tópicos escolares, nos permite fundamentar el diseño de una secuencia para ponerlas en conflicto, incluso como fuente de inspiración de preguntas estructurantes o paradigmáticas iniciales. Por ello, nos hemos documentado sobre las concepciones alternativas descritas en la literatura didáctica que sobre tópicos relativos a erosión, formación de rocas, aguas subterráneas, ciclo del agua, transporte de sedimentos, litoral, playas, arena, etc. Como no podía ser de otra manera, Pedrinaci (2000) es de obligada referencia, tanto en la búsqueda de concepciones del alumnado en Geología (origen de las rocas, inmutabilidad del relieve, construcción de montañas, tiempo geológico, etc.) como orientaciones didácticas sobre Geología. Francek (2013) realiza una revisión de más de quinientas concepciones alternativas en todos los niveles educativos, como la idea de que las placas tectónicas están hechas de "arena". En un trabajo previo con otros colegas Nelson, Aron y Francek, (1992, citados por Francek, 2013) ya incidieron sobre las ideas de los niños ante el movimiento del agua y la erosión y la formación de rocas, en cómo los niños consideran que el agua siempre fluye en una dirección (hacia abajo) sin reconocer las corrientes del agua para explicar el fenómeno de las olas. En cuanto a la formación de las rocas Kusnick (2002, citado por Francek, 2013) aporta la idea de los estudiantes de que no relacionan la erosión con la formación de nuevas rocas y arena de playa. En este sentido, Martínez, Bannan y Kitsantas, (2012, citados por Francek, 2013), comenta que el alumnado de primaria cree que la erosión solo ocurre mientras la lluvia está cayendo y no relacionan que el material de roca de arena formó parte alguna vez de la capa sólida de la Tierra. Dickerson y Dawkins (2004) y Roca, Márquez y Sanmartí (2013) indicaron que la mayoría de los niños no reconocen la existencia de las aguas subterráneas, y los que sí suelen creer que el agua se encuentra contenida debajo de tierra en compartimentos estanco.

\section{Secuencia de actividades de indagación basada en modelos (MBI).}

Al diseñar una secuencia de indagación, además de poner en conflicto las concepciones alternativas antes descritas, relacionadas con la estructura y formación de la playa (Nombela, 2005; Cárdenes y Santillán, 2015), como por ejemplo, el proceso de degradación y regeneración (Eirexas, Jiménez-Aleixandre y Gutiérrez-Roger, 2009), debemos tener en cuenta que el enfoque de enseñanza se convierte en un fin en sí 
mismo pues también les hacemos vivir cómo "se hace y se habla" ciencias centrándonos en la búsqueda de pruebas y en el uso de modelos que sirvan para explicar y predecir.

Esta secuencia sería idónea de realizar en la playa pero también puede ser adaptada para una o dos sesiones en el aula, con alumnado del tercer ciclo de Primaria. Hemos elegido este curso pues en el currículo oficial, en los estándares de aprendizaje se indica: "Reconoce y explica algunos ecosistemas: pradera, charca, bosque, litoral y ciudad, y los seres vivos que en ellos habitan" (MECD, 2014).

Toda secuencia de indagación parte de una pregunta que da sentido a la misma, cercana a los estudiantes, que les engancha: "¿Por qué el mar no se desparrama si solo tiene arena alrededor?"

Esta pregunta puede derivar en dos vertientes: “¿Por qué la arena del mar contiene el agua y no se dispersa?" $Y$, “¿cómo es posible que hagamos un hoyo en la playa y encontremos agua?"

En la siguiente tabla exponemos la secuencia de actividades comentada junto con la idea principal (1a columna) y la fase del enfoque de enseñanza de indagación basada en modelos (2 2 columna).

Tabla 1. Secuencia indagación por modelos con actividades comentadas

\begin{tabular}{|c|c|c|c|}
\hline Idea & Fase & Actividades & Comentarios y expectativas \\
\hline \multirow[t]{2}{*}{$\begin{array}{l}\frac{\pi}{\pi} \\
\frac{\pi}{2} \\
\frac{\pi}{0} \\
\frac{0}{0} \\
\frac{0}{0} \\
\frac{1}{0} \\
\frac{1}{0} \\
0 \\
0\end{array}$} & 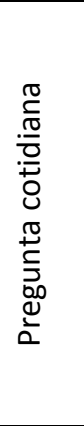 & $\begin{array}{l}\text { A1. ¿Qué creéis que hay en la arena? } \\
\text { ¿De qué creéis que está formada? }\end{array}$ & $\begin{array}{l}\text { En el caso de encontrarnos en el aula } \\
\text { podemos usar la Figura } 2 \text { como inicio, } \\
\text { junto con una bolsa llena de arena de } \\
\text { playa para que puedan manipularla, } \\
\text { observar de qué está compuesta y si esta } \\
\text { observación mejora cuando usamos la } \\
\text { lupa. } \\
\text { En la puesta en común incidiremos en si } \\
\text { identificamos restos animales (conchas } \\
\text { pequeñas), microplásticos, etc. }\end{array}$ \\
\hline & 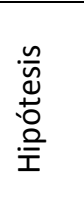 & $\begin{array}{l}\text { A2. ¿Cómo se ha formado la arena? } \\
\text { ¿Por qué algunas "piedrecillas" son } \\
\text { más grandes y otras más pequeñas? } \\
\text { Anotad en vuestro papel las } \\
\text { respuestas. }\end{array}$ & $\begin{array}{l}\text { En estas dos primeras actividades pueden } \\
\text { surgir concepciones alternativas } \\
\text { habituales de no asociar el proceso de } \\
\text { erosión con la formación de rocas. }\end{array}$ \\
\hline
\end{tabular}




\begin{tabular}{|c|c|c|c|}
\hline Idea & Fase & Actividades & Comentarios y expectativas \\
\hline & 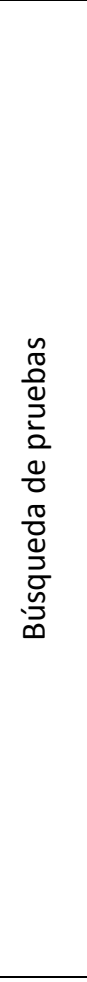 & $\begin{array}{l}\text { A3a. ¿Cómo podemos comprobar que } \\
\text { nuestras hipótesis sobre la formación } \\
\text { de la arena son ciertas? } \\
\text { A3b. En caso de que no haya } \\
\text { propuestas por parte de los } \\
\text { estudiantes, podemos proponer: } \\
\text { Los materiales necesarios serán una } \\
\text { lata o recipiente vacío, piedras, } \\
\text { conchas, metal y tiza. } \\
\text { En primer lugar, meter los distintos } \\
\text { elementos en el recipiente y cerrar } \\
\text { bien la tapa. Agitar rápidamente } \\
\text { durante un rato. Abriremos el } \\
\text { recipiente y se mirará en el interior, } \\
\text { veremos una pequeña capa de polvo } \\
\text { en el fondo y fragmentos encima. } \\
\text { Extenderemos el resultado de la } \\
\text { agitación sobre un recipiente plano y } \\
\text { veremos que en el polvo hay algo de } \\
\text { piedras, conchas y tiza, mientras que } \\
\text { hay trocitos de piedra más pequeña } \\
\text { que se han formado de las grandes } \\
\text { por el golpeo. }\end{array}$ & $\begin{array}{l}\text { Por diversos motivos las rocas se golpean } \\
\text { unas con otras y se desprenden pequeños } \\
\text { pedazos que a su vez chocan y chocan } \\
\text { dividiéndose cada vez más hasta quedar } \\
\text { en la forma de arena o polvo. A este } \\
\text { fenómeno se lo conoce como erosión. } \\
\text { El hecho de haber incluido tiza en el } \\
\text { experimento, aunque ésta no se presente } \\
\text { de tal manera en el ecosistema playa, es } \\
\text { que sepan diferenciar que según los } \\
\text { distintos elementos que se encuentren y } \\
\text { de la manera en que se rozan, agitan o } \\
\text { golpean se fragmentarán de una manera } \\
\text { u otra, produciendo polvo o pequeñas } \\
\text { piedrecitas. A partir de aquí, ya entra en } \\
\text { juego el concepto de erosión. Debido a la } \\
\text { agitación del recipiente los objetos } \\
\text { comenzaron a chocarse unos con otros, } \\
\text { esto provocó que algunos trocitos de } \\
\text { cada material se fueran desprendiendo y } \\
\text { acumulándose en el fondo. }\end{array}$ \\
\hline \multirow[t]{2}{*}{ 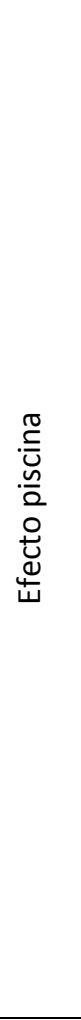 } & 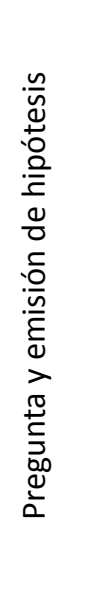 & $\begin{array}{l}\text { A4a. Una vez que hemos explicitado } \\
\text { de qué está hecha la arena y cómo se } \\
\text { forma, volveremos a la pregunta que } \\
\text { estructura la secuencia: ¿Por qué el } \\
\text { mar no se desparrama si solo tiene } \\
\text { arena alrededor? } \\
\text { A4b. Realiza el dibujo de una playa y } \\
\text { de una piscina. Anota las diferencias } \\
\text { de los elementos naturales o } \\
\text { artificiales que sujetan el agua. }\end{array}$ & $\begin{array}{l}\text { El objetivo es reconocer que en la piscina } \\
\text { el agua está contenida por una estructura } \\
\text { impermeable y en la playa el agua se } \\
\text { encuentra rodeada por arena que es } \\
\text { permeable. } \\
\text { En este punto crucial de la secuencia } \\
\text { esperamos dos tipos de respuestas: unos } \\
\text { que indiquen que la arena y la piscina son } \\
\text { iguales (no hay agua bajo la arena de la } \\
\text { playa) y otros que, tal vez, sí pinten agua } \\
\text { bajo la arena de la playa. Por lo tanto, es } \\
\text { importantísimo que justifiquen sus } \\
\text { hipótesis (en su experiencia, o por su } \\
\text { conocimiento, etc.). }\end{array}$ \\
\hline & 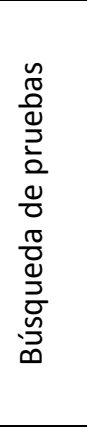 & $\begin{array}{l}\text { A5a. ¿Qué diseño podemos plantear } \\
\text { para comprobar si la arena se } \\
\text { comporta como una piscina o es } \\
\text { permeable? } \\
\text { A5b. ¿Podemos construir un castillo } \\
\text { de arena seca? ¿Cómo es posible que } \\
\text { la arena seca no nos sirva para } \\
\text { construir castillos y si está húmeda sí } \\
\text { puede utilizarse para su } \\
\text { construcción? }\end{array}$ & $\begin{array}{l}\text { Ambas ideas son complementarias por lo } \\
\text { que, si los estudiantes tienen dificultades } \\
\text { podemos plantearles los siguientes } \\
\text { diseños experimentales: } \\
\text { Para la contención por arena: por grupos } \\
\text { de } 3 / 4 \text { alumnado van a tener un cubo de } \\
\text { playa y dos montones de arena. } \\
\text { El objetivo de esta actividad es la } \\
\text { necesidad de mojar la arena para que se } \\
\text { compacte. }\end{array}$ \\
\hline 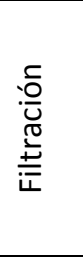 & 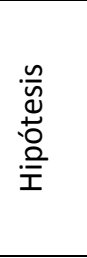 & $\begin{array}{l}\text { A6. Si hacemos un hoyo a dos metros } \\
\text { de distancia de la orilla, encontramos } \\
\text { agua, ¿cómo creéis que ha llegado el } \\
\text { agua hasta ahí? Explicadlo con un } \\
\text { dibujo. }\end{array}$ & $\begin{array}{l}\text { Para la filtración, corrientes de aguas } \\
\text { subterráneas y nivel freático podemos } \\
\text { plantear esta pregunta. Ello pondrá en } \\
\text { conflicto lo que Dickerson y Dawkins } \\
\text { (2004) indicaron que los niños no } \\
\text { relacionan el agua superficial con las }\end{array}$ \\
\hline
\end{tabular}




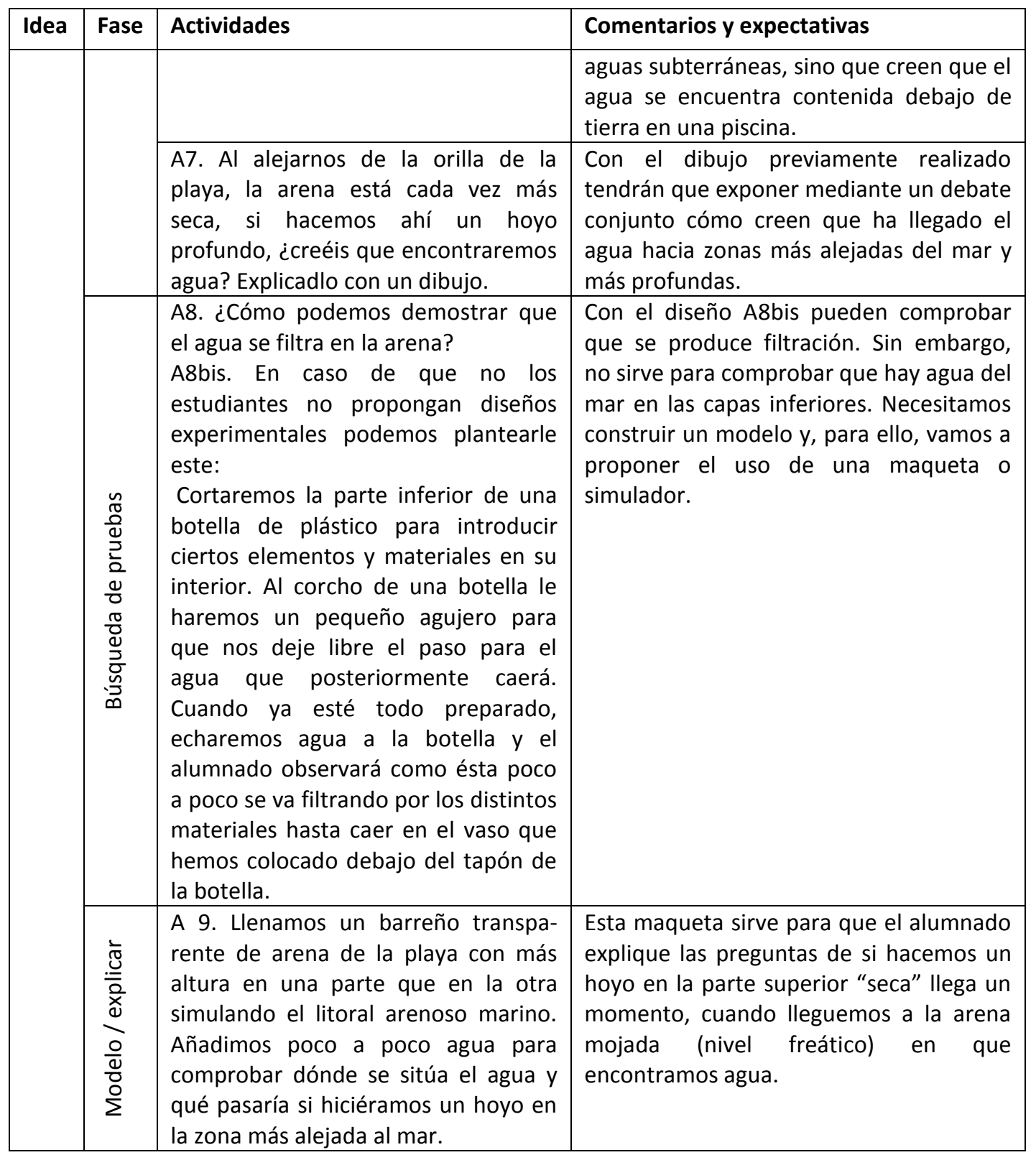

La secuencia de actividades propuesta podrá ser aplicada por el profesorado de Primaria con mayor o menor grado de apertura según sus intereses y lo habituado que esté el alumnado en realizar actividades de indagación. Tras la aplicación de esta secuencia se puede proponer una evaluación que incida en el reconocimiento por parte del alumnado de lo que ha aprendido y cómo se ha sentido realizándola (Jiménez-Liso, Romero, Martínez-Chico, Amat y Salmerón, 2017), de manera que incluya tanto el reconocimiento del aprendizaje de contenidos científicos como de la competencia en indagación (Ferrés, Marbá y Sanmartí, 2015).

\section{A modo de conclusión}

Los educadores ambientales, al igual que el profesorado en general, que quieren implementar enfoques de enseñanza alternativos al enfoque tradicional, a menudo se topan con las dificultades de no haber vivido o de encontrar secuencias de actividades fundamentadas en la investigación didáctica. En este artículo hemos partido de la 
insatisfacción por las típicas actividades realizadas en la playa (limpieza de residuos, salida litoral para observar especies o sus huellas, etc.) para plantear una secuencia de actividades que pongan en conflicto las principales concepciones alternativas del alumnado, descritas en la literatura.

La secuencia de actividades de indagación por modelo sobre el agua del mar y la arena, permite a los educadores reflexionar sobre la finalidad de la actividad, sobre la ambientalización y contextualización del currículo escolar, sobre el qué enseñar en educación ambiental $y$, fundamentalmente, plantea un enfoque de enseñanza transferible y con sentido.

\section{Agradecimientos}

Este trabajo ha sido posible por la cofinanciación de los proyectos EDU2015-69701-P y P11-SEJ-7355 (@Sensociencia) y por el contexto del máster interuniversitario de Educación Ambiental y de AGata Verde.

\section{Referencias bibliográficas}

Ahijado, C., Uranga, I., Vázquez, R. y Yenes, M. (2001). Ecoauditoría Escolar. Madrid: Consejería de Educación.

Cárdenes, V. y Santillán Pedrosa, E. (2015). Propuesta de actividad didáctica para el estudio del color, tamaño y composición de arenas de playa. Un caso práctico en la costa de Bélgica. Enseñanza de las Ciencias de la Tierra, 23(3), 315-319.

Chawla, L. (2006). Research Methods to Investigate Significant Life Experiences: review and recommendations. Reprinted from Environmental Education Research (1998) 4 (4), pp. 383-397. Environmental Education Research, 12(3-4), 359-374. http://doi.org/10.1080/1350462980040403

Couso, D. (2014). De la moda de "aprender indagando" a la indagación para modelizar: una reflexión crítica. En M. A. De las Heras (coord.). Investigación y transferencia para una educación en ciencias: un reto emocionante. Huelva: Servicio de Publicaciones de la Universidad de Huelva. Recuperado de: http://uhu.es/26edce/actas/docs/conferencias/pdf/26ENCUENTRO_DCEConferenciaPlenarialnaugural.pdf

Dickerson, D. y Dawkins, K. (2004). Eighth Grade Students' Understandings of Groundwater. Journal of Geoscience Education. 178-181.

Eirexas, F., Jiménez-Aleixandre, M.P. y Gutiérrez-Roger, X. (2009). Uso de datos, construcción de argumentos y evaluación de actuaciones sobre la degradación del litoral. Enseñanza de las Ciencias, número extra, VIII Congreso Internacional sobre Investigación en Didáctica de las Ciencias, Barcelona, 2129-2135. http://ensciencias.uab.es/congreso09/numeroextra/art-2129-2135.pdf

Ferrés, C., Marbà, A. y Sanmartí, N. (2015). Trabajos de indagación de los alumnos: instrumentos de evaluación e identificación de dificultades. Revista Eureka sobre Enseñanza y Divulgación de las Ciencias, 12(1), 22-37. DOI: 10498/16922

Francek, M. (2013). A Compilation and Review of over 500 Geoscience Misconceptions. International Journal of Science Education, 35(1), 31-64. http://dx.doi.org/10.1080/09500693.2012.736644

Jiménez-Liso, M.R., Romero, M., Martínez-Chico, M., Amat, A. y Salmerón, E. (2017). 
Sensopíldora chicles Orbit: uso de sensores para promover prácticas científicas de indagación con modelos. Enseñanza de las Ciencias, núm. Extra, 685-690.

López-Gay, R., Jiménez-Liso, M.R. y Martínez-Chico, M. (2015). Enseñanza de un modelo de energía mediante indagación y uso de sensores. Alambique, 80, 38-48.

Martínez del Águila, Y., Montoro, A.B., Jiménez-Liso, M.R. y Gil, F. (2016). Perfiles de profesores de secundaria en formación inicial con relación a la química cotidiana. Educación Química, 27(2), 143-153. https://doi.org/10.1016/j.eq.2015.11.003

MECD (2014). Real Decreto 126/2014, de 28 de febrero, por el que se establece el currículo básico de la Educación Primaria. Recuperado de: https://www.boe.es/buscar/pdf/2014/BOE-A-2014-2222-consolidado.pdf

Nombela, M.A. (2005). ¡Vamos a la playa! Dinámica sedimentaria en playas. Enseñanza de las Ciencias de la Tierra, 13(2), 138-147.

Pedrinaci, E. (2000). La enseñanza y el aprendizaje del conocimiento geológico. En Perales, F.J. y Cañal, P. (coords.). Didáctica de las Ciencias Experimentales. Teoría y práctica de la enseñanza de las ciencias. Alcoy: Marfil, 479-504.

Pujol, R.M. (2003). Didáctica de las ciencias en la educación primaria. Madrid: Ed. Síntesis.

Rebelo, D., Marques, L. y Costa, N. (2004).Actividades en ambientes exteriores al aula en la Educación en Ciencias: contribuciones para su operatividad. Enseñanza de las Ciencias de la Tierra, 19(1), 15-25.

Roca, M., Márquez, C. y Sanmartí, N. (2013). Las preguntas de los alumnos: Una propuesta de análisis. Enseñanza de las Ciencias, 31(1), 95-114.

Rodríguez, F. y García, E. (2009). El activismo que no cesa. Obstáculos para incorporar la metodología didáctica basada en la investigación del alumno a la práctica de la Educación Ambiental. Investigación en la Escuela, 67, 23-36.

Sanmartí, N. y Márquez, C. (2017). Aprendizaje de las ciencias basado en proyectos: del contexto a la acción. Ápice. Revista de Educación Científica, 1(1), 3-16. https://doi.org/10.17979/arec.2017.1.1.2020

Sauvé, L. (2010). Educación científica y educación ambiental: un cruce fecundo. Enseñanza de las Ciencias, 28 (1), 5-18. 